\title{
DIGIPRESCRIPTION: AN INTELLIGENT SYSTEM TO ENABLE PAPERLESS PRESCRIPTION USING MOBILE COMPUTING and Natural-Language Processing
}

\author{
Richard Zhang ${ }^{1}$, Mary Zhao ${ }^{1}$, Yucheng Jiang ${ }^{2}$, \\ Sophadeth Rithya ${ }^{2}$ and Yu Sun ${ }^{2}$ \\ ${ }^{1}$ Irvine High School. 4321 Walnut Ave Irvine, CA 92604 \\ ${ }^{2}$ California State Polytechnic University, Pomona, CA, 91768
}

\begin{abstract}
Through our app, it is aimed to teach and tell the patients how to use the drug properly taking off the chances of putting their lives in danger, especially the elderly. It is also efficient to give patients these instructions as well as saving lots of paper. Because of the law, every drug that is given from the pharmacy to the user includes a receipt that lists information of, patient's information, drug information, insurance information, directions on taking the medicine (black box warning issued by FDA), medication details on how it works, side effects, storage rules, and etc. These pieces of information are crucial to patients, where it tells them how to use the drug properly, but most people would throw these receipts away, which is a risk as well as a waste. Through using this app, the patient can efficiently get information on how to properly use the drug. This application is also helpful, where the user can choose to set reminders on when to eat this drug each week or month.
\end{abstract}

\section{KEYWORDS}

Reminder System, HTML, Python, Firebase, Bootstrap

\section{INTRODUCTION}

Visiting a doctor's office and getting a prescription for drugs is not a rare occurrence in the modern world countries. Many patients will go through the process of visiting a doctor, get a prescription, go to a drugstore, and get some medicine to treat their disease. Prescription, abbreviated $\mathrm{Rx}$, is an instruction written by a doctor authorizing the provision of medicines or treatments to patients. The 2016 National Ambulatory Medical Care Survey shows that the total number of physician office visits in the United States is 883.7 million. In the doctor's office, the doctor collects the patient's information such as heart rate, weight, blood pressure, and height before reaching a conclusion of how to treat the patient. Many patients need to employ prescription drugs to treat their disease. To prescribe drugs for the patient, the doctor will write a handwritten prescription paper or send the prescription electronically to the pharmacy. In the case of handwritten prescriptions, the patient will receive a prescription paper guiding them on what medicine to buy and how to consume medicine. This prescription contains information including:

- The patient's name the date of birth, and the address of the patient.

- Name of the drug, amount to be consumed every time, and frequency of taking the drug.

David C. Wyld et al. (Eds): ACITY, DPPR, VLSI, WeST, DSA, CNDC, IoTE, AIAA, NLPTA - 2020 
- Amount of the drug that the pharmacy gives the patient and the number of refills.

- The signature of the doctor and physician identifies like NPI and DEA number.

The patient needs to bring the handwritten prescription paper with him to a pharmacy or drug store in order to get the prescription drugs recommended by the doctor. At the pharmacy, the pharmacist communicates with the patient on what medicine the patient can have and how much the drug will cost. The patient will decide which drug to choose depends on the doctor's recommendation. Once the decision has been made, the patient picks up the prescription drug at the pharmacy.

Handwritten prescription paper creates inconvenience for the patients and wastes paper resources. The standard size of the handwritten prescription paper is 4 inches by 5.5 inches. Such a size of paper is inconvenient to the patients. It does not fit into the pocket, and putting the paper in a bag might damage the prescription. The paper prescription also has the risk to be lost. When the patients damage or lose their prescription, they have to run back to the doctor's office for the same prescription. This causes unnecessary trouble for patients. Another issue of handwritten prescription is paper usage. An average of 4 billion retail prescriptions is filled every year in the United States. This enormous amount of paper used is very costly. The cost is both financially and environmentally. The doctor has to buy more when he runs out of prescription paper. To the environment, producing paper means deforestation, or cutting down trees. 93 percent of paper produced comes from trees, and $42 \%$ of trees harvested is used to make paper. Caused by deforestation, the future agricultural productivity in tropical regions is threatened by the increase in average temperature and related extreme temperatures, as well as the decrease in average rainfall and rainfall frequency.

We use an online database to store the prescription information and a mobile app for the patients to access. Utilizing the technologies of HTML [1] coding, fire store database, and flutter coding, we created an application software addressing the issues on the inconvenience and paper waste of handwritten prescription paper. Our app is composed of an online website for the doctor to use, a cloud database to store the data, and a mobile app for the patients to use. The website functions to receive the prescription information that the doctor enters rather than handwriting on paper. A QR code containing that information will be generated from the website and shown on the screen. The patient then uses the mobile app installed on their phone to scan the QR code that contains their prescription information. This information will be stored in our online database. If the patients sign in with their account, they could see their past scanned prescription information history. To obtain the prescription drug, the patients just need to carry their phones with them to the pharmacy. Our method differs from the traditional handwritten prescription paper by not having the patients carry the prescription paper with them, but only a mobile app to be installed on their phone. This approach is more convenient since most people today already carry a mobile phone with them wherever they travel. The patients do not have to worry about losing the prescription as they can access the stored information with their account. Also, our app helps to reduce paper waste because our app eliminates the usage of handwritten prescription paper.

The rest of this paper is organized as follows: Section 2 details the challenges we encountered in the process of experimenting and designing samples. Section 3 focuses on the details of the solutions corresponding to the challenges mentioned in Section 2. Section 4 introduces the relevant details of our experiments, and then Section 5 introduces related work. Finally, Section 6 gives conclusions and points out the future work of the project.

\section{Challenges}

There are a few of challenges existing in the project. They will be discussed in this section. 


\subsection{Challenge 1: New Drugs Update}

The system needs to know how to update itself due to the new drugs that come out each year. Through the research online, it shows that twenty to twenty-five new drugs are made per year for the past two decades. When each new drug comes out, the app needs to know how to update itself, possibly through another website online. It will be difficult for an individual managing the app, to keep updating the site throughout the year. For example, if a new drug was invented and tested that would help cure the COVID-19, the app would be able to add a new regulation with this drug by itself, possibly through another website's newly added information in its database, and add this information onto our app. When this new information is added to our app's database, the patient would be able to scan the QR Code, given from the pharmacy, and get the right information on how to use this drug correctly.

\subsection{Challenge 2: Create QR Codes by Pharmacy}

The Pharmacy needs to have our app's program in order to create QR codes. In order for the pharmacy to create a QR Code for the patient to scan, they would have to input our app's program into their computer. Our program has to also be compatible with the program the pharmacy is using in order for them to input their information of the drug, creating the QR Code, and sending it for the patient to use. A pharmacy always has its own system to work with a patient, giving them the right drugs. Our app has to be compatible with the pharmacy's software, be able to create a recognizable barcode and be able to print onto the label. This is a compatible challenge, where there are several pharmacies' software's which are used by different pharmacies. So, the real question is, how can we make our app compatible with all pharmacies' software?

\subsection{Challenge 3: Check OTC Drugs}

The app should be able to check OTC drugs. The OTC stands for "over the counter" which represents the people that buy drugs and medicine without the guidance of the doctor's prescriptions. If the patient is not buying a drug from the doctor's prescription, the app can tell the patient if the drug is safe or not to buy. When the patient goes to the store, he can scan the drug or input drug name, then the app would tell the patient if the drug is safe or not for the buyer to buy. The app would be able to tell the patient if the drug is compatible or not to the buyer's statistics of allergies, or problems. The app would also need to connect to the NDC system which stands for "the drug code of commercial". This is difficult to be completed due to the need to add more information into the database of the app. This may lead to us thinking of all the possibilities that could occur, depending on the patient's conditions.

\section{Solution}

\subsection{Overview of the Solution}

Because of the law, each medicine purchased by the patient comes with a receipt with the patient's information, drug information, insurance information, directions on taking the medicine (black box warning issued by FDA), medication details on how it works, side effects, storage rules, and etc. Most people after receiving medicine from a pharmacy, would throw away the receipt, which could be a factor of putting their lives in danger, as well as wasting lots of paper. On the pharmacy's end, they can use the app to create a QR Code for the patient to scan, containing the long list of information that would be on the receipt. For the patients, they would use the app to scan the QR Code, giving them the list of all the medicine's information, as well as 
a way to set reminders on when to take the medicine. There are several similar tools at present [12][13]. For this app, you would first need to sign up for an account. After you signed up, you have to sign in to your account. After you signed in, this page will include your profile, your drug prescription history, and the button to scan QR Codes. Each prescription listed in that main page will have the drug number, the drug name, and the date the drug was added. Each drug page will have the information from the receipt, with the choice of reading the information out loud, as well as the choice of adding reminders on when to take this drug.

\subsection{Implementation of the App}

The web end of our app is designed for the use of doctors and pharmacists. The prescriber is using our website can deliver their medical instructions to patients more conveniently. On our website, a form is provided for the prescriber to fill out with patient information. After entering the necessary information, the prescriber can click on the "submit" button to generate a QR code for the patient to scan. This QR code contains all the information entered by the doctor. Patients that scanned the QR code will be able to access the information anytime in our mobile app.

\begin{tabular}{l} 
Full Name \\
Enter Full Name \\
Date of Birth \\
mm/dd/yyy \\
Male O Female \\
Search For Drug \\
Drug Name \\
Drug Name \\
Drug Description \\
Enter Drug Description \\
Instruction \\
\hline Submit \\
\hline
\end{tabular}

Figure 1: submission page for the app

We implemented this website using languages HTML and Python [2] [3]. At the top of our website is a navbar created from Bootstrap [7][8][9]. The main body of our website is a list of text fields for the prescriber to input patient information. We decided to incorporate the patient's full name, date of birth, gender, drug name, drug description, and doctor instruction in our form. Each input is labeled to guide doctors filling in the patient information properly. We limited input type to improve information accuracy. For example, the input type of date of birth is limited to date. 


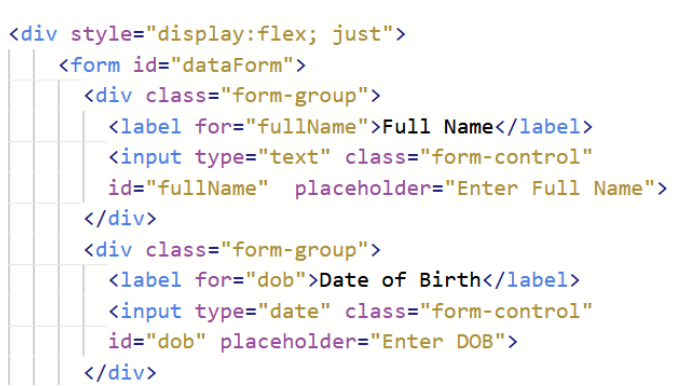

Figure 2: HTML for the app

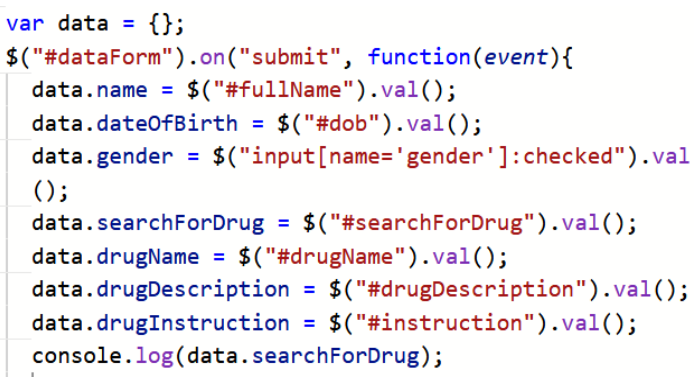

Figure 3: Data processing for the app

In the JS phase of our application, we created a variable database to contain all the information entered by the prescriber. The "submit" button in our website has an on-pressed function of collecting data. After the doctor filled in all the patient information and pressed the "submit" button, the string of data in each input will be collected, named, and stored in the database.

To improve the website's user friendliness, we added the auto complete function for the prescriber to search for a medicine. Suggestions of existing drugs pop out as the prescriber enters the drug name letters. This function operates by matching the entered drug name with existing drugs information stored in our second database.

Our drug information in the second database is obtained by the method web scraping. Beautiful Soup and html parser were used during our web scraping. Using Python, we targeted specific tag "li" of data from our source website. All the pieces of data including the targeted tag on the source website will be detected and printed out to our second database. After web scraping is finished, our second database contains names and information links of many existing drugs.

\subsection{Functionalities of the App}

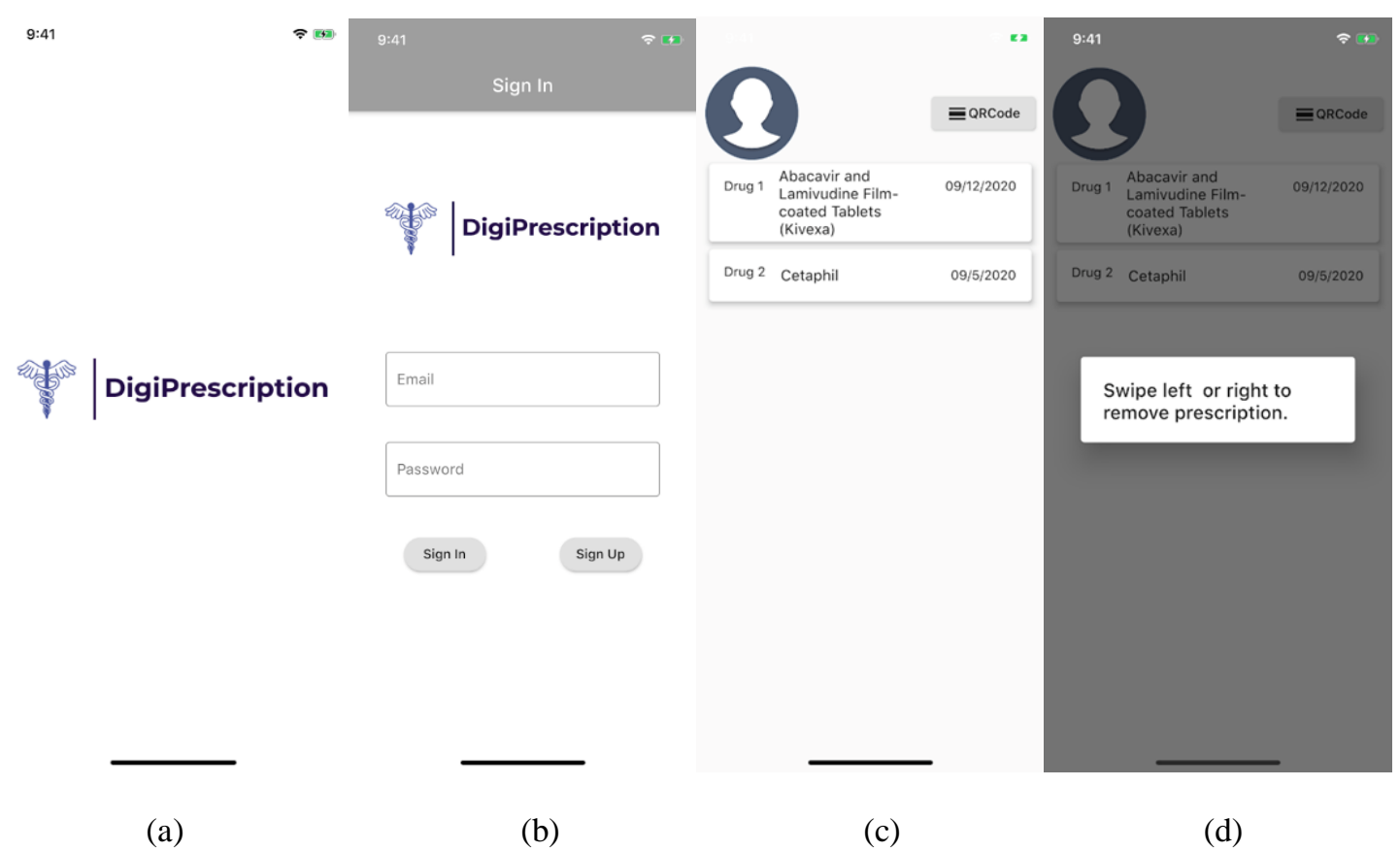

Figure 4: screenshot of the app 
Figure 4 (a) is the title page, which includes the logo I created, and the sign-in and sign-up page Figure 4 (b). If you haven't created an account, then you would press the Sign-Up page and then you would have to re-sign in. I linked my app to firebase [4][5][6] so that when a user creates an account, the information of the user would be stored there.

After you created an account for the app, this page would show up, displaying your profile with all your previous prescriptions. It would also have an option to scan QR Codes to add more prescriptions to the list. For each drug prescription displayed Figure 4 (c), there would be the drug number, the drug name, and the date when this drug prescription was added to your account. When a drug prescription is no longer needed or when you are done using the drug, you can swipe right or left to delete the prescription Figure 4 (d).

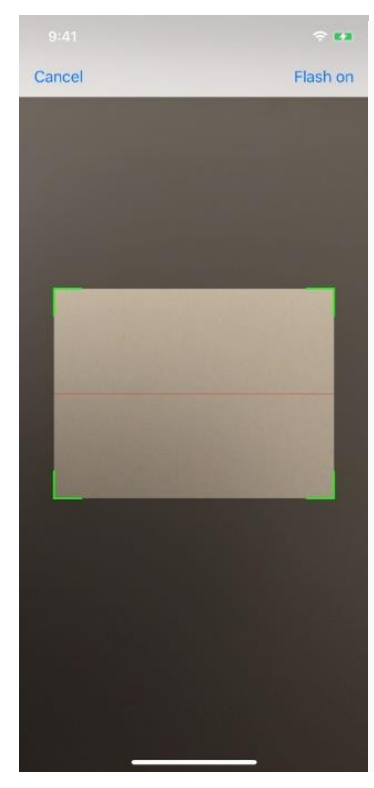

(a)

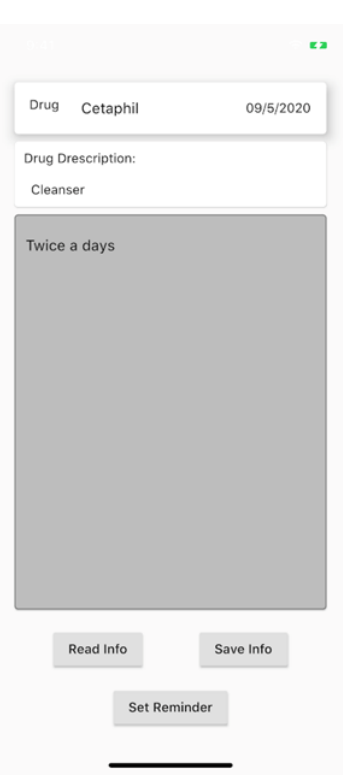

(b)

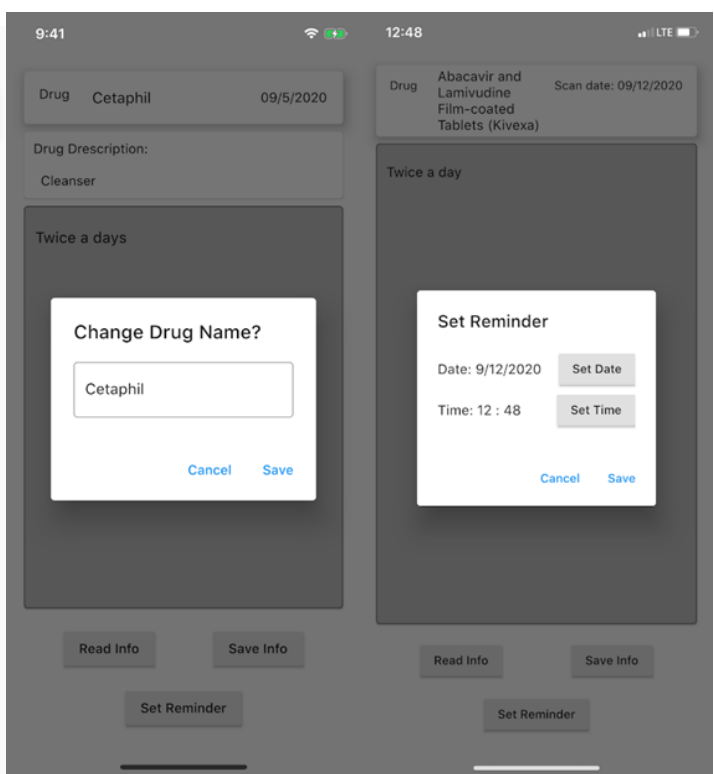

(c) (d)

Figure 5: screenshot of the app

With this feature, you can scan the barcode provided on your drug bottle. After you scan the QR Code, the Prescription Details page will show up, giving you all the information from the receipt.

\subsection{Prescription Details}

After you have scanned the QR Code on the drug bottle, you will get this page with all the information from the receipt. This will include the patient's information, drug information, insurance information, directions on taking the medicine (black box warning issued by FDA), medication details on how it works, side effects, storage rules, and etc. This page will also have the ability to read the information out loud as well as setting reminders of when to take the medicine.

In the Prescription Details Page, other than having the information from the receipt, there is also the choice of changing the name of the drug prescription as well as setting the date of reminders. For setting the dates of taking the medicine, you can choose the time each day and the days per month. 


\section{EXPERIMENTS}

To evaluate the effectiveness of our app in helping patients, we collected user evaluation and feedback from 30 patients who used the app.

We collected evaluations from the participants by asking them to list out the things that they liked and/or disliked about the app. Some things that the patients liked about the app were features such as the reminder and prescription details. Some participants reported that they can retrieve information such as drug information, insurance information, directions on taking the medicine, side effects, storage rules anytime they want.

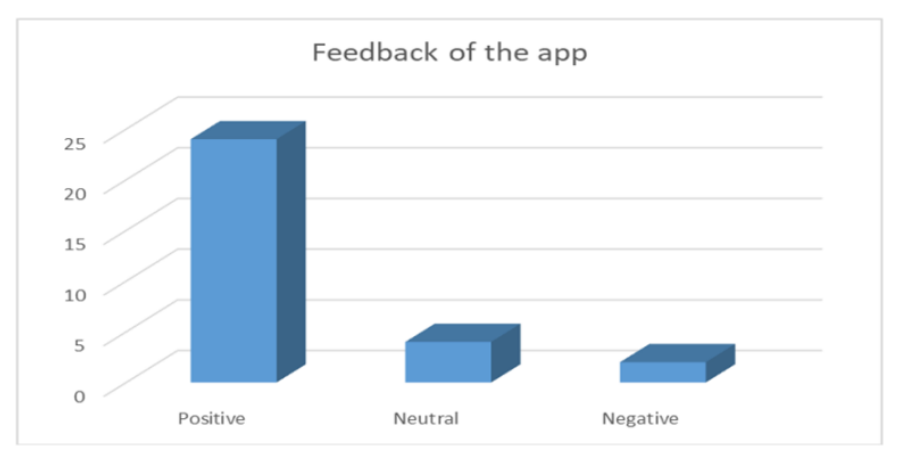

Figure 6: feedback collected from patients

Lastly, we asked the patients to give any feedback or suggestions for the app in terms of improvements of the features in the future. We believe that all of these suggestions are extremely valuable to the future improvements of the app.

\section{RELATED WORK}

A medication reminder mobile app: does it work for different age ranges? Mina Fallah, Mobin Yasini designed an Android based software that reminds patients with chronic disease to consume their routine medication. The app was developed and evaluated based on its userfriendliness, efficacy, and usability. An inquiry of 2 efficiency questions and 10 usability questions was created to assess the app. Through this app, medication consumers, app users, and patient relatives are joined together. Patient's adherence to the medication improves due to the connection between doctor and patients. Our app differs from this app in the employment of QR code. Doctors enter medication information on our web page to generate a QR code for the patients to scan with their phone. This process of making a reminder is more convenient.

Medication Reminder \& Healthcare is an android application, developed by Deepti Ameta, Kalpana Mudaliar and Palak Patel [10], which provides various functions for user patients. Their app allows interaction between doctors and patients. Patients can search for a doctor and make appointment disease wise or based on their location. Patients are also able to search for disease information through their app. Our app varies from this app with our QR code scan and online database. Patients' scanned information will be stored online, and they are able to access it by logging in with their account.

Slagle, J.M., Gordon, J.S., Harris, C.E., Davison, C.L., Culpepper, D.K., Scott P. and Johnson, K.B., (2011) "MyMediHealth - Designing a next generation system for child-centered medication management" [11]. This medication reminding app was developed for children, allowing parents to create an intuitive 24 hours day planner, which meals and medications can be 
instructed in each time interval. This app contains advanced features such as auto-population function (allows medication to be taken multiple times in a day) and medication-specific business logic (safety limit on the amount of medication taken in a period of time). Our app differs from this app with the applicant of QR code and ability to read drug description. After entering the drug description, users can tap on the "read info" button to have the app read description of the drug.

\section{CONClusion ANd Future Work}

Through creating this application, lots of good effects have come out of this. We have saved a lot of paper but also making it easier for patients to know the instructions on how to take the drug properly without putting their lives in danger. The patient can also have the choice to set reminders on when they are going to take the medicines per week and per month, which is also very efficient.

A limitation within this app is translation. Even though the app can read the information from the receipt out loud for minorities, it is a problem for people that don't know English. Another limitation is that for people that are elders, the app might be hard for them to use the app due to technological advancements. It is going to be hard for them to know how to use this app. The last limitation is that the application should know when the drug is used up due to the time when the patient added the drug to the list. This function will significantly improve compliance which will have a good outcome on medication therapy and clinical results.

For translating, and adding a solution for compliance problems in pharmacy, we can add more features to this application for this to happen. For the elders that don't know how to use this app, we will try to do experiments and surveys seeing how to make this app easier to use through the data we receive.

\section{REFERENCES}

[1] Lemay, Laura, and Arman Revised By-Danesh. Teach yourself web publishing with HTML 4 in a week. Sams, 1997.

[2] Grinberg, Miguel. Flask web development: developing web applications with python. " O'Reilly Media, Inc.", 2018.

[3] Dolgert, A., L. Gibbons, and V. Kuznetsov. "Rapid web development using AJAX and Python." In Journal of Physics: Conference Series, vol. 119, no. 4, p. 042011. IOP Publishing, 2008.

[4] Moroney, Laurence, Moroney, and Anglin. Definitive Guide to Firebase. Apress, 2017.

[5] Moroney, Laurence. "Firebase Cloud Messaging." In The Definitive Guide to Firebase, pp. 163-188. Apress, Berkeley, CA, 2017.

[6] Stonehem, Bill. Google Android Firebase: Learning the Basics. Vol. 1. First Rank Publishing, 2016.

[7] Spurlock, Jake. Bootstrap: Responsive Web Development. " O'Reilly Media, Inc.", 2013.

[8] Shenoy, Aravind, and Ulrich Sossou. Learning Bootstrap. Packt Publishing Ltd, 2014.

[9] Radford, Stephen. Learning Web Development with Bootstrap and AngularJS. Packt Publishing Ltd, 2015. 
[10] Ameta, Deepti, Kalpana Mudaliar, and Palak Patel. "Medication reminder and healthcare-an android application." International Journal of Managing Public Sector Information and Communication Technologies (IJMPICT) Vol 6 (2015).

[11] Slagle, Jason M., Jeffry S. Gordon, Christopher E. Harris, Coda L. Davison, DeMoyne K. Culpepper, Patti Scott, and Kevin B. Johnson. "MyMediHealth-Designing a next generation system for childcentered medication management." Journal of biomedical informatics 43, no. 5 (2010): S27-S31.

[12] Herrmann, James M., Gerald S. Indorf, and Sunway R. Wang. "Interactive medication reminder/dispenser device." U.S. Patent 5,805,051, issued September 8, 1998.

[13] Hawley, Tarwa L., and Jon H. Kirk Jr. "Medication reminder." U.S. Patent 6,325,534, issued December 4, 2001.

(C) 2020 By AIRCC Publishing Corporation. This article is published under the Creative Commons Attribution (CC BY) license. 